\title{
Left-Handed Coplanar Waveguide
}

\author{
Jan Macháč, Martin Hudlička, Ján Zehentner, Nikolaos G. Spiliotis", Abbas S. Omar ${ }^{*}$ \\ Czech Technical University, Technicka 2, 16627 Prague 6, Czech Republic \\ *University of Magdeburg, PF 4120, 39016 Magdeburg, Germany
}

\begin{abstract}
This paper proposes a new version of a left-handed coplanar waveguide. The line has a fully uniplanar structure without vias and lumped elements. The series capacitors are represented by interdigital capacitors and the parallel inductors by short circuited CPW stubs connected to a ground metallization. The measured frequency dependences of $S_{21}$ and $S_{11}$ agree well with the data predicted by simulation. The simple equivalent circuit of the line unit cell was set up by fitting its dispersion characteristic to the dispersion characteristic of the first left-handed mode calculated by the CST Microwave Studio.
\end{abstract}

Index Terms - Coplanar waveguide, metamaterial, lefthanded transmission line.

\section{INTRODUCTION}

Left-handed (LH) materials, or metamaterials, have become a reality, and they have been demonstrated to be practically realizable [1]-[3]. These metamaterials have unique properties that might be used for various applications, e.g., in antennas, hybrid ring couplers, phase shifters, ideal lenses, anomalous refractors and reflectors, single mode waveguides, very short resonators exhibiting zeroth order resonance, frequency selective surfaces and perfect magnetic layers. These materials can be designed as transmission lines [2] periodically loaded by proper inclusions to get the left-handed properties or in a volume version based on the system of split ring resonators and straight wires periodically placed in space [1], [3]. Planar structures are of special interest in the applications in microwave circuits [4], namely if they do not contain any lumped elements [5]-[7].

This paper presents the design of a uniplanar left-handed coplanar waveguide (LHCPW) without vias or lumped elements. The layout modifies the known structure [5] and makes it more compact. The presented line aims at circuit applications, whereas the CPW in [5] was utilized for a leaky wave antenna design. The CPW in [7] works with split ring resonators, and due to their resonant behaviour it is narrowband. Our LHCPW was designed, manufactured and measured. The propagation of a LH wave, or a backward wave, is shown in the frequency band depending on the line parameters. The propagation of the higher order LH modes is documented by simulations done in the CST Microwave Studio (MwS). The measured frequency dependent scattering parameters compare well with the S-parameters calculated by MwS. The equivalent circuit of the LHCPW unit cell is derived by fitting its dispersion characteristic to the dispersion characteristic of the unit cell calculated by MwS.

\section{LAYOUT OF THE LEFT-HANDED COPLANAR WAVEGUIDE}

The idea of the LHCPW comes from the equivalent circuit of the LH transmission line [2], which incorporates series capacitors and parallel inductors together with a hosting transmission line. The layout of the unit cell of the proposed LHCPW is shown in Fig. 1, together with its simple equivalent circuit. The series capacitor is represented by an interdigital capacitor and the parallel inductor by shortcircuited CPW stubs connected to the ground metallization. The remaining equivalent circuit elements will be described in section IV. All these elements are, however, frequency dependent. Consequently, the equivalent circuit of the line cell [4] must be correspondingly modified. By periodically translating the cell shown in Fig. 1 we get the layout of the LHCPW shown in Fig. 2. The first specimen of the line was

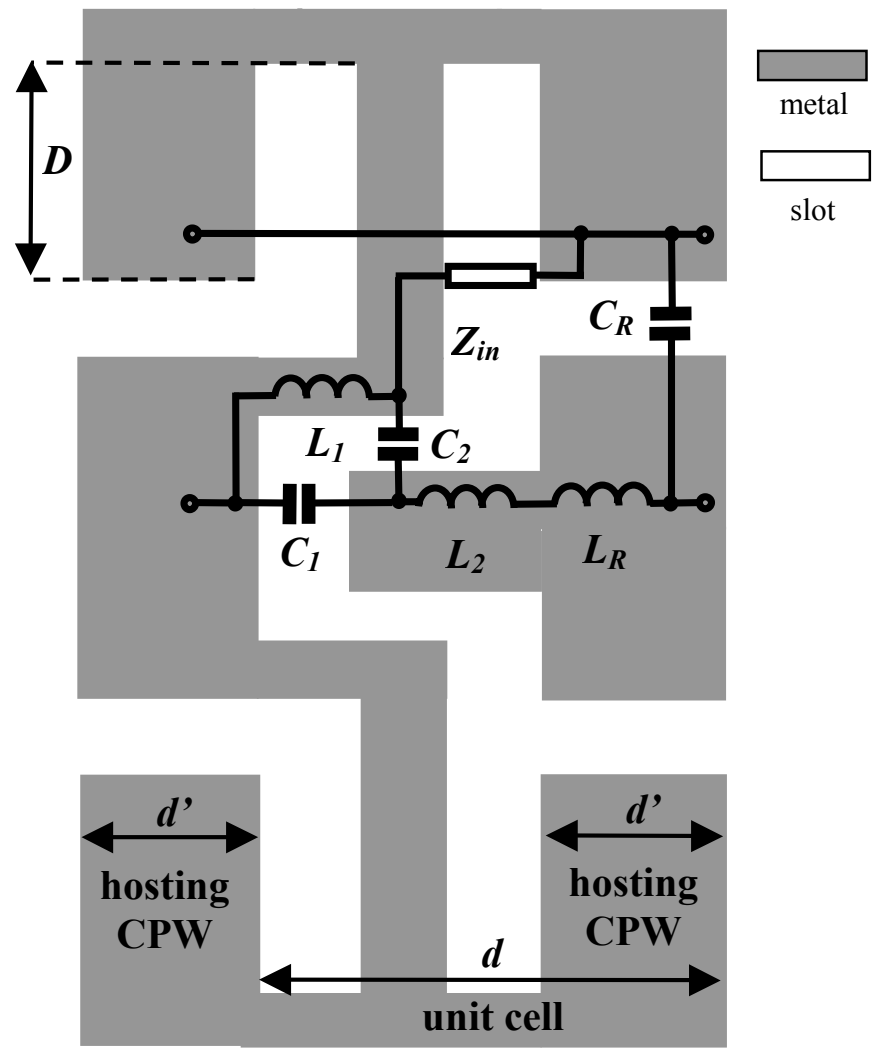

Fig. 1 The unit cell of the LHCPW. 
designed using a ROGERS RO4003C substrate with permittivity $3.38,0.813 \mathrm{~mm}$ in thickness and metallization $0.035 \mathrm{~mm}$ in thickness.

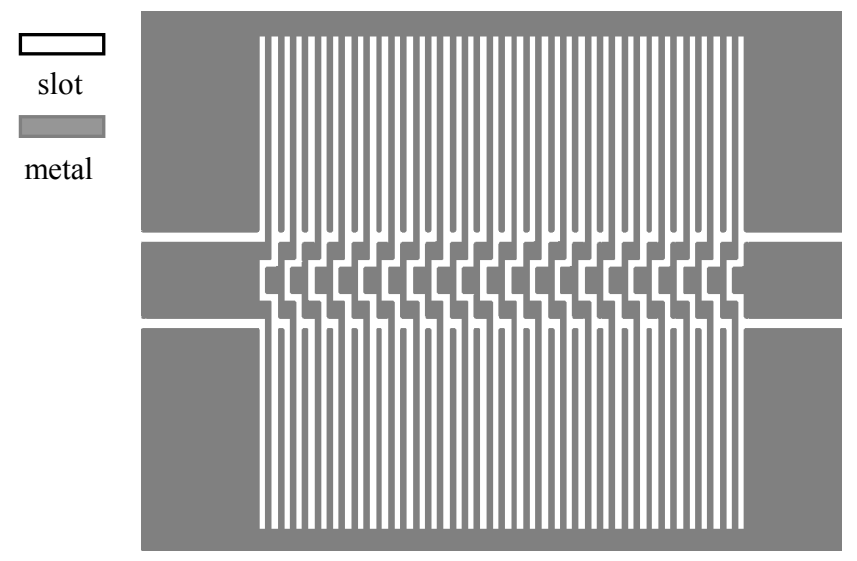

Fig. 2 Layout of the LHCPW.

\section{LHCPW CHARACTERISTICS}

The dispersion diagram of modes propagating along LHCPW shown in Fig. 2 calculated by the CST Microwave Studio [8] is plotted in Fig. 3. Each branch of this dispersion characteristic defines a pass-band in which the LHCPW

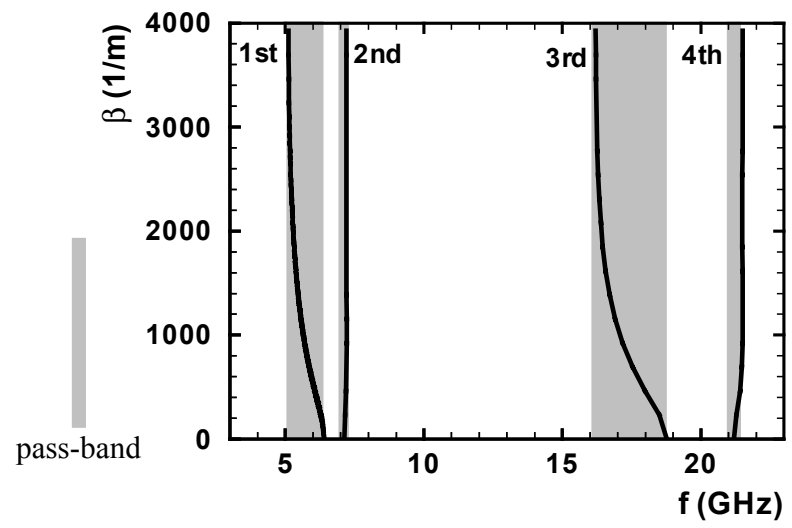

Fig. 3 Dispersion characteristics of the four lowest modes on the LHCPW defined in the text above.

transmits a particular mode. These modes have different field distributions, shown in Fig. 4. The first and the second modes are of the first order from the viewpoint of the field distribution along the CPW stubs, as their field reaches only one maximum here. The third and the fourth modes are, consequently, higher order modes, as their field twice reaches a maximum along the CPW stubs. The first and the third modes are LH modes, as their phase constant decreases with frequency. The second and the fourth branches of the dispersion characteristic are two-valued and three-valued functions of frequency, not distinctly seen in Fig. 3. Their corresponding pass-bands are very narrow. If ever the modes that are physical within the second and fourth pass-bands propagate, they will propagate as right-handed $(\mathrm{RH})$ modes, as their phase constant dominantly increases with frequency. We pay attention only to the first LH mode propagating at lowest frequencies. The visualization of the field of this mode, by the CST Microwave Studio, shows the wave propagating along the RHCPW sections from the input towards the output port, while on the LHCPW section the wave runs in the opposite direction. The phase constant of this mode decreases with frequency and the wavelength increases.

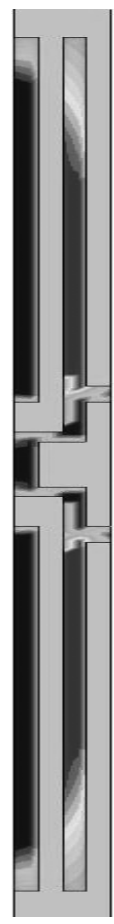

1 st

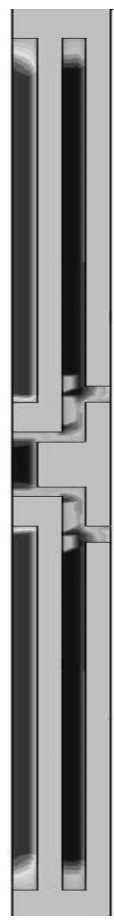

2nd

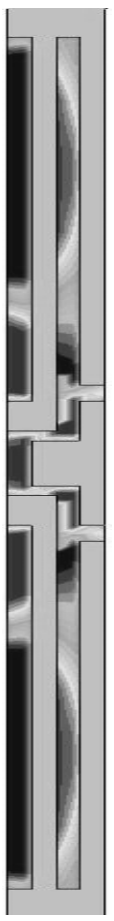

3 rd

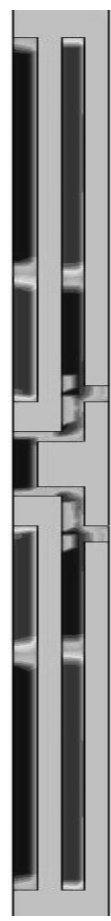

4th
Fig. 4 Distribution of an electric field component parallel to the CPW axis, Fig. 2, calculated within the surface with the metallization of the LHCPW unit cell shown in Fig. 2. The distribution is plotted for the four modes corresponding to the four pass-bands shown in Fig, 3.

The frequency dependence of the modulus of the scattering parameter $S_{21}$ calculated by $\mathrm{MwS}$ is plotted in Fig. 5. Its comparison with $S_{21}$ measured in the pass-band of the first

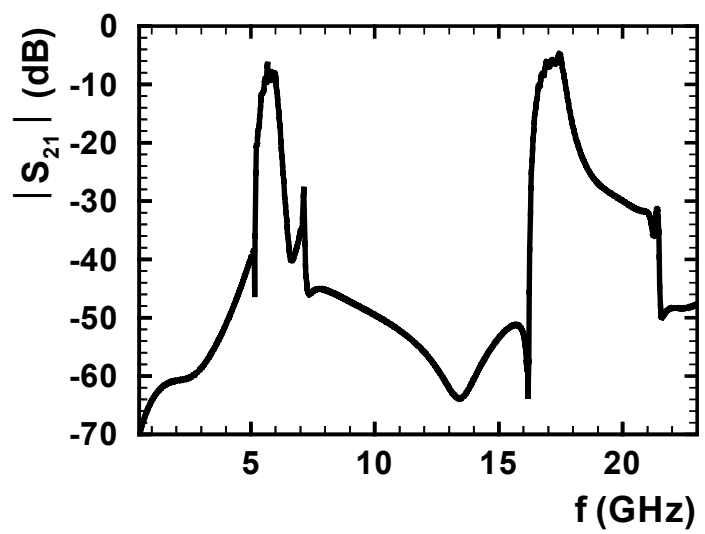

Fig. 5 Calculated modulus of $S_{21}$ of the manufactured LHCPW 


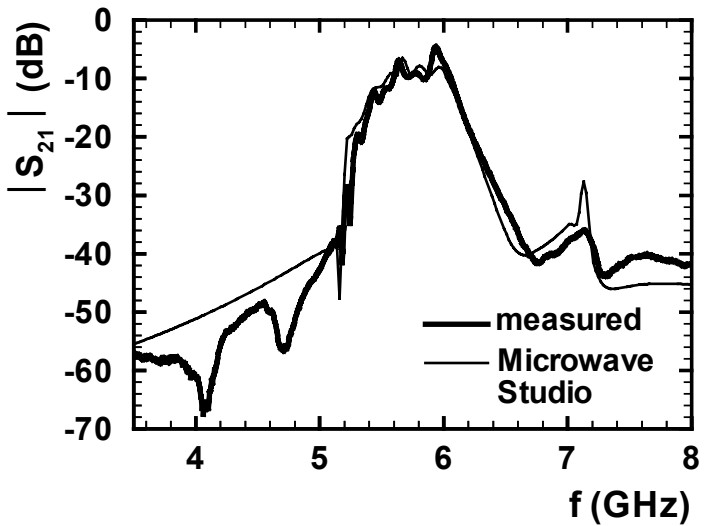

Fig. 6 Measured and calculated modulus of $S_{21}$ of the manufactured LHCPW.

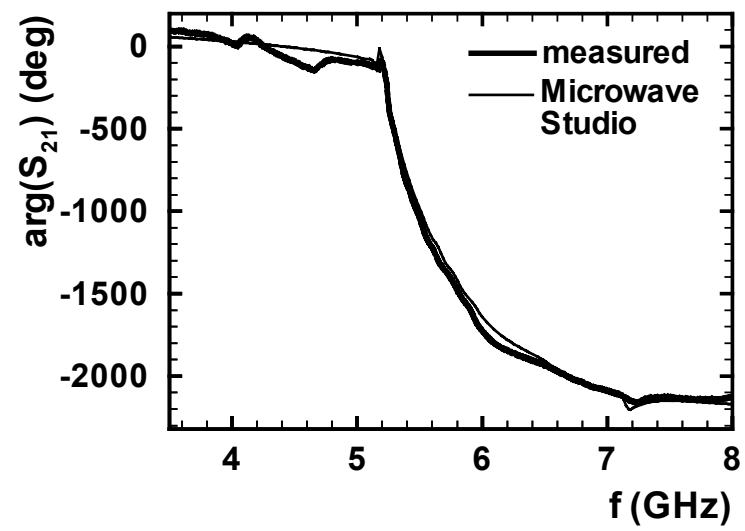

Fig. 7 Measured and calculated phase of $S_{21}$ of the manufactured LHCPW.

LH mode is shown in Fig. 6. The unwrapped phase of $S_{21}$ is plotted in Fig. 7. The measured records fit the calculated patterns well. The pass-bands shown in Fig. 5 correspond to those shown in Fig. 3. The first left-handed mode can propagate from 5.1 to $6.4 \mathrm{GHz}$. The practical transmission band of this left-handed mode is however narrower, from about 5.4 up to $6.2 \mathrm{GHz}$, i.e., about $13 \%$ of the middle frequency. The peak of the frequency characteristic, in Figs. 5 and 6 at $7.2 \mathrm{GHz}$, corresponds to the propagation of the second mode. The third mode, which is left-handed, can propagate between 16.2 and $18.8 \mathrm{GHz}$, as seen in Fig. 3. This band is reduced from above by the fast decrease of $S_{21}$ to 17.8 $\mathrm{GHz}$, Fig. 5. The pass-band of the fourth mode from 21.1 to 21.5 GHz, in Fig. 3, corresponds to a latent peak at $21.2 \mathrm{GHz}$ in the frequency characteristic of $S_{21}$ shown in Fig. 5.

\section{LHCPW EQUIVALENT CIRCUIT}

The equivalent circuit of the LHCPW unit cell is shown in Fig. 1. This circuit contains two inductors $L_{1}, L_{2}$ and two capacitors $C_{1}, C_{2}$. Additional elements $L_{R}, C_{R}$ represent the hosting $\mathrm{CPW}$ and are determined by its characteristic impedance $Z_{0}$ and the propagation constant $k$

$$
Z_{0}=\sqrt{L_{R}^{\prime} / C_{R}^{\prime}}, \quad k=\omega \sqrt{L_{R}^{\prime} C_{R}^{\prime}}
$$

where (') denotes the value taken per unit length. Impedance $Z_{\text {in }}$ represents the input impedance of two short-circuited CPW stubs with finite-extent ground planes of length $D$ connected in parallel, which is

$$
Z_{\text {in }}=\mathrm{j} Z_{0 e} \tan \left(\beta_{0} D\right) / 2,
$$

where $Z_{0 e}$ is the characteristic impedance of the even mode on the CPW with finite-extent ground planes [9], and $\beta_{0}$ is the phase constant of this mode. In our case, the stub length is $D=7 \mathrm{~mm}$ and $Z_{0 e}=148.6 \Omega$ and $Z_{\text {in }}$ is inductive. An ideal lefthanded transmission line consists only of series capacitor $C_{1}$ and the parallel inductor formed by the impedance $Z_{\text {in }}$. Parasitic series inductors $L_{1}, L_{2}$ and parallel capacitor $C_{2}$ have been added to the model of the LHCPW layout to make it more realistic. The effect of coupling between neighbouring cells is not taken into account. The equivalent circuit is proposed to model the dispersion characteristic of the first left-handed mode.

It is not possible to make a direct calculation of an ABCD matrix and to derive the dispersion characteristic of the circuit shown in Fig. 1. This circuit is therefore rearranged to the modified equivalent circuit shown in Fig. 8, where the hosting CPW is $d$ ' in length and $d$ is the length of the unit cell, Fig. 1. The circuit elements $Z_{s 1}, Z_{s 2}$ and $Y_{p}$

$$
Z_{s 1}=\frac{L_{1} / C_{1}}{\mathrm{j} \omega L_{1}+1 /\left(\mathrm{j} \omega C_{1}\right)+1 /\left(\mathrm{j} \omega C_{2}\right)},
$$

$$
Z_{s 2}=\frac{-1 /\left(\omega^{2} C_{1} C_{2}\right)}{\mathrm{j} \omega L_{1}+1 /\left(\mathrm{j} \omega C_{1}\right)+1 /\left(\mathrm{j} \omega C_{2}\right)}+\mathrm{j} \omega L_{2},
$$

$Y_{p}=\left(\frac{L_{1} / C_{2}}{\mathrm{j} \omega L_{1}+1 /\left(\mathrm{j} \omega C_{1}\right)+1 /\left(\mathrm{j} \omega C_{2}\right)}+\mathrm{j} Z_{0 e} \tan \left(\beta_{0} D\right)\right)^{-1}$,

are obtained by the star-delta transformation of elements $L_{1}$, $C_{1}, C_{2}$, Fig. 1, and by some additional circuit rearrangements.

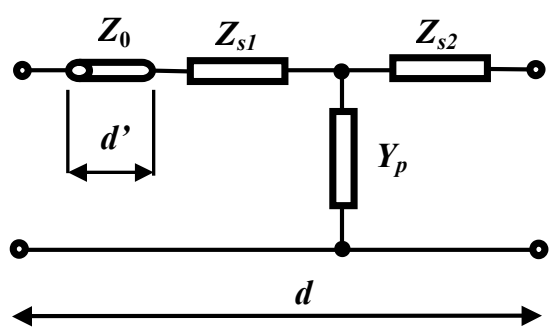

Fig. 8 Modified equivalent circuit of one cell of the LHCPW used in the calculation of the dispersion characteristic.

The dispersion characteristic is thus derived using the modified equivalent circuit of the LHCPW unit cell shown in Fig. 8. The ABCD matrix of this circuit is calculated. Then 
applying Floquet's theorem the dispersion characteristic of the LHCPW is obtained

$$
\begin{aligned}
& \cos (\beta d)=\frac{1}{2}\left(2+Y_{p}\left(Z_{s 1}+Z_{s 2}\right)\right) \cos \left(k d^{\prime}\right)+ \\
& +\frac{\mathrm{j}}{2 Z_{0}}\left(Z_{s 1}+Z_{s 2}+Y_{p}\left(Z_{0}^{2}+Z_{s 1} Z_{s 2}\right)\right) \sin \left(k d^{\prime}\right) .
\end{aligned}
$$

The values of the lumped-elements from Fig. 1 were determined by fitting the dispersion characteristic defined by (5) to the dispersion characteristic from Fig. 3 calculated by MwS. The resulting values are $L_{1}=9.56 \mathrm{nH}, C_{1}=0.195 \mathrm{pF}$, $L_{2}=5.25 \mathrm{nH}$ and $C_{2}=0.88 \mathrm{pF}$.

A comparison of the dispersion characteristic calculated by MwS, the characteristic calculated using (6), and the measured characteristic is shown in Fig. 9. The equivalent circuit is quite simple, but it very accurately represents the LHCPW in the frequency band of the first LH mode propagation. At higher frequencies the proportions of the circuit become comparable with the wavelength, and the equivalent circuit gradually losses validity.

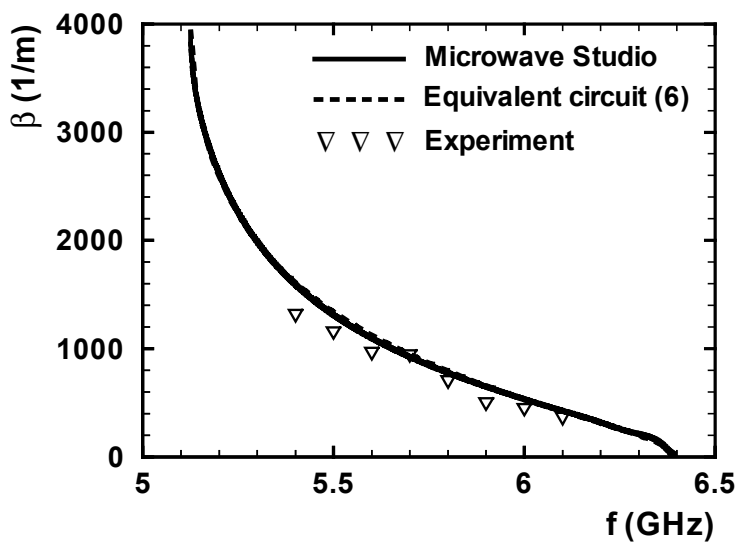

Fig. 9 Comparison of the dispersion characteristics of the fabricated line calculated by the CST Microwave Studio, calculated by means of the equivalent circuit, Figs. 1 and 8, (6), and measured.

\section{CONCLUSION}

A new version of the left-handed coplanar waveguide was designed, fabricated and measured. The line has a fully planar structure without lumped elements and vias. The basic lefthanded mode propagates in a frequency band about $0.8 \mathrm{GHz}$ in width. The propagation of the higher order left-handed modes was presented by calculations. The dispersion characteristic calculated by the CST Microwave Studio predicts the passbands of the left-handed and right-handed modes, which correspond well with the calculated and measured transmission characteristics. The dispersion characteristic of the lowest LH mode was verified by measurement. The simple lumped equivalent circuit of the LHCPW was set up by fitting its dispersion characteristic to the dispersion characteristic calculated by the CST Microwave Studio. This circuit models the LHCPW in the pass-band of the lowest LH mode.

\section{ACKNOWLEDGEMENT}

This work has been supported by the Grant Agency of the Czech Republic under project 102/03/0449 "New circuit devices for communication technology" and the experiments were done with support from the Ministry of Culture in Sachsen-Anhalt, Germany.

\section{REFERENCES}

[1] D. R. Smith, W. J. Padilla, D. C. Vier, S. C. Nemat-Nasser, S. Schultz, "Composite medium with simultaneously negative permeability and permittivity", Pys. Rev. Lett., Vol. 84, No. 18, May 2000, pp. 4184-4187.

[2] C. Caloz, T. Itoh, "Application of the transmission line theory of left-handed ( $\mathrm{LH})$ materials to the realization of a microstrip LH transmission line", IEEE-APS International Symposium, San Antonio, TX, June 2002, Proc. Vol. 1, pp. 412-415.

[3] R. W. Ziolkowski, C-Y. Cheng, "Tailoring double negative metamaterial responses to achieve anomalous propagation effects along microstrip transmission lines", 2003 IEEE MTT-S Digest, Vol. 1, June 2003, Philadelphia, pp. 203-206.

[4] C. Caloz, T. Itoh, "Novel microwave devices and structures based on the transmission line approach of meta-materials", 2003 IEEE MTT-S Digest, Vol. 1, June 2003, Philadelphia, USA, pp. 195-198.

[5] A. Grbic, G. V. Eleftheriades, "Leaky CPW-based slot antenna arrays for millimeter-wave application", IEEE Trans Antenna Propag., Vol. AP-50, No. 11, Nov. 2002, pp. 1494-1504.

[6] A. Sanada, K. Murakami, S. Aso, H. Kubo, I. Awai, "A viafree microstrip left-handed transmission line", 2004 IEEE MTT-S Digest, Vol. 1, June 2004, Fort Worth, TX, pp. 301304.

[7] F. Falcone, F. Martín, J. Bonache, R. Marqués, T. Lopetegi, M. Sorola, "Left handed coplanar waveguide band pass filters based on bi-layer split ring resonators", Microwave and Wireless Components Letters, Vol. 14, No. 1, January 2004, pp. 10-12.

[8] D. R. Smith, D. C. Vier, N. Kroll, S. Schultz, "Direct calculation of permeability and permittivity for a left-handed metamaterial", Applied Physics Letters, Vol. 77, No. 14, Oct. 2000, pp. 2246-2248.

[9] G. Ghione, C. U. Naldi, "Coplanar Waveguides for MMIC Applications: Effect of Upper Shielding, Conductor Backing, Finite-Extent Ground Planes, and Line-to-Line Coupling", IEEE Trans. Microwave Theory Tech., vol. MTT-35, March 1987, pp. 260-267. 